Original Article

\title{
Isolated Mitral Valve Repair in Patients with Reduced Left Ventricular Ejection Fraction
}

\author{
Thilo Noack, MD, ${ }^{*}$ Mateo Marin Cuartas, MD, ${ }^{*}$ Philipp Kiefer, MD, Jens Garbade, MD, PhD, \\ Bettina Pfannmueller, MD, PhD, Joerg Seeburger, MD, PhD, and Michael A. Borger, MD, PhD
}

\begin{abstract}
Purpose: This study aims to analyze the clinical outcomes after isolated mitral valve (MV) repair in patients with reduced left ventricular ejection fraction (LVEF $<50 \%)$ with focus on perioperative characteristics, survival, and freedom from reoperations. Methods: Between 1997 and 2015, 557 patients with reduced LVEF (age: 62.8 \pm 11.7 years, male: 320) underwent MV repair for symptomatic mitral regurgitation (MR). Etiologies were dilated non-ischemic cardiomyopathy and ischemic cardiomyopathy in 487 (87.4\%) and $70(12.6 \%)$ patients, respectively; these were classified into three different subgroups: LVEF 40\%-49\% (group 1), 30\%-39\% (group 2), and <30\% (group 3).

Results: Overall, 294, 145, and 118 patients had an LVEF of 40\%-49\%, 30\%-39\%, and $<30 \%$, respectively. Logistic EuroSCORE was significantly higher $(P<0.001)$ as the LVEF worsened. The survival analysis for groups 1-3, respectively, revealed the following: 30-day mortality: $1.4 \%, 3.4 \%$, and 7.6\% $(P<0.001)$; 1-year survival: 93.9\%, 89.4\%, and 82\% $(P<0.001)$; 5-year survival: 81.2\%, 75.2\%, and 58\% $(P<0.001)$.

Conclusion: MV repair in patients with impaired LVEF could be performed safely with good clinical short- and mid-term outcome. Nevertheless, reduced preoperative LVEF correlates with worse perioperative and long-term survival.
\end{abstract}

Keywords: mitral valve, mitral regurgitation, mitral valve repair, heart failure

\section{Introduction}

Heart failure (HF) is an established poor prognostic factor in patients undergoing mitral valve (MV) repair for mitral regurgitation (MR). ${ }^{1,2}$ Recently, a European analysis reported that $50 \%$ of patients with severe symptomatic

University Department of Cardiac Surgery, Heart Center Leipzig, Leipzig, Germany

Received: April 4, 2019; Accepted: July 1, 2019

Corresponding author: Thilo Noack, MD. University Department of Cardiac Surgery, Heart Center Leipzig, Struempellstrasse 39, 04289 Leipzig, Germany

Email: thilo.noack@medizin.uni-leipzig.de

*Contributed equally to this work

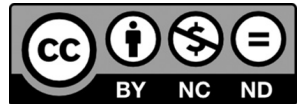

This work is licensed under a Creative Commons Attribution-NonCommercialNoDerivatives International License.

(C)2019 The Editorial Committee of Annals of Thoracic and Cardiovascular Surgery
MR were considered ineligible for surgical MV interventions primarily because of advanced age, HF, and concomitant comorbidities, ${ }^{3)}$ highlighting the need for more evidence in patients with the decreased left ventricular ejection fraction (LVEF) and symptomatic MR. HF due to ischemic or dilated cardiomyopathies causes secondary MR - a ventricular problem more than valvular dysfunction per se. ${ }^{2,4)}$ In patients with secondary MR, the mitral subvalvular apparatus and leaflets are predominantly normal but left ventricular dysfunction and dilatation cause eccentric displacement of the papillary muscles ${ }^{2)}$; this along with annular dilatation causes leaflet tethering and decreased valve coaptation, in turn causing MR. Reportedly, the resulting MR causes volume overload, fibrosis, and deleterious LV remodeling to an already decompensated LV. ${ }^{2,4)}$ Such changes correlate with poor outcomes without any therapeutic intervention, ${ }^{1,4)}$ and early treatment of precursors could decrease the mortality rate. ${ }^{5)}$ This complex pathophysiological mechanism with more 
extensive involvement of the heart as a whole illustrates why outcomes after MV repair in patients with secondary MR are more disappointing, as isolated valve repair does not correct ventricular dysfunction and only addresses one component of the disease.

MV surgical repair techniques, typically used for secondary MR, primarily aim to actively remodel the dilated annulus with a rigid annuloplasty ring. Although additional interventions to enhance valve coaptation, such as secondary or tertiary chordal cutting, as well as papillary muscle sling, have been reported, these have not been extensively applied. ${ }^{6}$

However, with time, the definition of HF has changed, and understanding of pathophysiological mechanisms has become more evident. The current HF guidelines enable a more precise classification of these patients depending on the LVEF degree. The current European Society of Cardiology (ESC) guidelines for the diagnosis and treatment of acute and chronic HF classified patients based on the following LVEF criteria: HF with reduced ejection fraction (HFrEF, LVEF < 40\%); HF with mid-range ejection fraction (HFmrEF, LVEF 40\%-49\%); and HF with preserved ejection fraction (HFpEF, LVEF $>50 \%$ ). ${ }^{7}$

The presence of MR and HF adversely affects decisionmaking as prognosis worsens, periprocedural risk elevates, and potential benefit from valve procedures declines. ${ }^{7)}$ The literature does not strongly support MV repair for secondary MR because of the incidence of residual and recurrent MR after MV repair. Additionally, latest guidelines for valvular heart disease consider MV surgery as a reasonable therapy only in patients with chronic severe secondary MR who are undergoing other concomitant cardiac procedures, such as bypass surgery or aortic valve (AV) replacement, or patients who are severely symptomatic (New York Heart Association [NYHA] classes III to IV) despite optimal medical therapy.,2) Reportedly, chordal-sparing MV replacement is a reasonable option to downsize annuloplasty repair. ${ }^{8-11)}$

However, to date, limited studies have evaluated the outcomes after MV repair based on different HF subgroups. In particular, HFmrEF (LVEF 40\%-49\%) remains a "grey zone," and its recognition as a separate group should be considered a new opportunity for research. Hence, this study aims to analyze the clinical outcomes after MV repair in patients with the reduced LVEF based on the LVEF subgroup classification with a focus on (1) perioperative characteristics, (2) survival, (3) risk factors for long-term survival, and (4) need of cardiac reoperations.

\section{Methods}

\section{Study cohort}

Between 1997 and 2015, 4178 consecutive patients underwent isolated MV repair with optional atrial septal defect (ASD) closure or cryoablation for atrial fibrillation (AFIB) at the Heart Center (Leipzig, Germany). Of these, we enrolled 557 patients (13.3\%) with LVEF < $50 \%$ in this study. Of note, patients with infective endocarditis, mitral stenosis, concomitant valve procedures, concomitant bypass surgery, aortic surgery, and surgical correction of congenital diseases were excluded from the analysis. All patients were categorized into three subgroups as follows: LVEF 40\%-49\% (group 1); LVEF 30\%-39\% (group 2); and LVEF <30\% (group 3). The primary endpoint was mid-/long-term survival and freedom from MV-related reoperation, whereas perioperative characteristics were the secondary endpoint. MV-related reoperation includes procedure-related reoperation (e.g., suture dehiscence, hemolysis, chordal shortening, incomplete repair) and valve-related reoperation (e.g., residual MR, degeneration). This study protocol was approved by the Ethics Committee of the medical faculty of the Leipzig University, and we obtained informed consent from all study participants.

\section{Surgical techniques}

Patients with contraindications for a minimally invasive approach ${ }^{12,13)}$ were operated through a median sternotomy, and the performance of the minimally invasive approach was based on the Leipzig minimally invasive MV valve technique. ${ }^{12,14)}$ Additionally, ring annuloplasty was used in all patients. Valve competency was tested with the water sealing probe; if the MV was found competent, the left atrium was de-aired and sutured. Furthermore, the MV was re-evaluated by intraoperative transesophageal echocardiography for residual MR after complete weaning of the cardiopulmonary bypass (CPB).

\section{Follow-up}

We followed up (mean postoperative follow-up: $5.9 \pm 4.5$ years) patients using either mailed questionnaires or phone contact with patients and/or family members, with supplemental information obtained from family physicians and referring cardiologists. The follow-up rate was $100 \%$ and was performed until the end of 2016. 
Table 1 Patients' baseline characteristics

\begin{tabular}{|c|c|c|c|c|c|}
\hline Variable & $\begin{array}{c}\text { Overall } \\
\text { LVEF }<50 \% \\
(n=557)\end{array}$ & $\begin{array}{c}\text { Group } 1 \\
\text { LVEF } 40 \%-49 \% \\
(n=294)\end{array}$ & $\begin{array}{c}\text { Group } 2 \\
\text { LVEF } 30 \%-39 \% \\
(n=145)\end{array}$ & $\begin{array}{c}\text { Group } 3 \\
\text { LVEF }<30 \% \\
(n=118)\end{array}$ & $P$ \\
\hline Mean age, years & $62.8 \pm 11.7$ & $63.5 \pm 12.2$ & $64.1 \pm 10.3$ & $59.5 \pm 11.6$ & 0.002 \\
\hline Male gender, $n(\%)$ & $320(57.4)$ & $167(56.8)$ & $77(53.1)$ & $76(64.4)$ & 0.173 \\
\hline BMI, $\mathrm{kg} / \mathrm{m}^{2}$ & $26.8 \pm 5.5$ & $25.7 \pm 5.0$ & $26.8 \pm 5.9$ & $25.8 \pm 6.2$ & 0.397 \\
\hline Diabetes mellitus, $n(\%)$ & $110(19.7)$ & $52(17.6)$ & $35(24.1)$ & $23(19.4)$ & 0.279 \\
\hline Hypertension, $n(\%)$ & $357(64)$ & $206(70.0)$ & $101(69.7)$ & $50(42.3)$ & $<0.001$ \\
\hline COPD, $n(\%)$ & $29(5.2)$ & $15(5.1)$ & $5(3.4)$ & $9(7.6)$ & 0.314 \\
\hline $\mathrm{PAD}, n(\%)$ & $29(5.2)$ & $18(6.1)$ & $6(4.1)$ & $5(4.2)$ & 0.589 \\
\hline $\mathrm{PHT}>60 \mathrm{mmHg}, n(\%)$ & $108(19.4)$ & $58(19.7)$ & $30(20.7)$ & $20(16.9)$ & 0.600 \\
\hline \multicolumn{6}{|l|}{ CAD } \\
\hline 1-Vessel, $n(\%)$ & $56(10.0)$ & $28(9.5)$ & $20(13.8)$ & $8(6.8)$ & \multirow{3}{*}{0.335} \\
\hline 2-Vessels, $n(\%)$ & $22(3.9)$ & $11(3.7)$ & $8(5.5)$ & $3(2.5)$ & \\
\hline 3-Vessels, $n(\%)$ & $14(2.5)$ & $7(2.4)$ & $5(3.4)$ & $2(1.7)$ & \\
\hline $\mathrm{AFIB}, n(\%)$ & $215(38.6)$ & $124(42.2)$ & $77(53.1)$ & $37(31.3)$ & 0.274 \\
\hline \multicolumn{6}{|l|}{ NYHA functional lass } \\
\hline $\mathrm{I} / \mathrm{II}, n(\%)$ & $250(44.9)$ & $174(59.2)$ & $56(38.6)$ & $20(16.9)$ & \multirow{3}{*}{$<0.00$} \\
\hline III, $n(\%)$ & $232(41.6)$ & $93(31.6)$ & $68(46.9)$ & $71(60.2)$ & \\
\hline $\mathrm{IV}, n(\%)$ & $75(13.5)$ & $27(9.2)$ & $21(14.5)$ & $27(22.9)$ & \\
\hline \multicolumn{6}{|l|}{ MR grade } \\
\hline Moderate-to-severe, $n(\%)$ & $23(4.1)$ & $13(4.4)$ & $6(4.1)$ & $4(3.3)$ & \multirow{2}{*}{0.893} \\
\hline Severe, $n(\%)$ & $534(95.9)$ & $281(95.6)$ & $138(95.9)$ & $114(96.6)$ & \\
\hline LVEF, \% & $36.8 \pm 9.4$ & $44.3 \pm 2.8$ & $33.7 \pm 2.8$ & $22.2 \pm 5.1$ & $<0.001$ \\
\hline \multicolumn{6}{|l|}{ Cardiomyopathy } \\
\hline Non-ischemic dilated, $n(\%)$ & $487(87.4)$ & $258(87.7)$ & $122(84.1)$ & $108(91.5)$ & \multirow{2}{*}{0.186} \\
\hline Ischemic, $n(\%)$ & $70(12.6)$ & $36(12.3)$ & $23(15.9)$ & $10(8.5)$ & \\
\hline Logistic EuroSCORE, \% & $7.2 \pm 5.7$ & $6.3 \pm 4.8$ & $7.4 \pm 6.0$ & $9.3 \pm 6.7$ & $<0.001$ \\
\hline
\end{tabular}

AFIB: atrial fibrillation; BMI: body mass index; CAD: coronary artery disease; COPD: chronic obstructive pulmonary disease; LVEF: left ventricular ejection fraction; MR: mitral regurgitation; NYHA: New York Heart Association; PAD: peripheral arterial disease

\section{Statistical analysis}

In this study, categorical variables are expressed as proportions, whereas continuous variables as mean \pm standard deviations. Normal distribution of continuous variables was tested with the Kolmogorov-Smirnov test. We compared categorical variables using the $\chi^{2}$ test or Fisher's exact test and continuous variables using the unpaired $t$-test. Additionally, survival and freedom from reoperation were analyzed with the Kaplan-Meier actuarial methods. Using the log-rank test, we performed survival and freedom from reoperation subgroup comparison. Risk factors for long-term survival were evaluated with multivariable Cox regression analysis. The appropriateness of variable transformations was determined by means of univariate analysis. Variables with a univariate $P<0.05$ or those with known clinical relevance but failing to meet this critical $\chi^{2}$ level were submitted to the multivariable models were submitted to the multivariable models. We considered $P<0.05$ as statistically significant. SPSS
Statistics Version 25.0 (SPSS, Inc., Chicago, IL, USA) was used to perform all statistical analyses.

\section{Results}

The subgroup analysis revealed that 294, 145, and 118 patients exhibited an LVEF of 40\%-49\%, 30\%-39\%, and $<30 \%$, respectively. Table 1 summarizes the preoperative baseline characteristics.

While right-sided mini-thoracotomy was the selected approach in 327 patients (58.7\%), a median sternotomy was performed in 230 (41.3\%) patients (Table 2). A minimally invasive technique was more frequently selected in patients with a better LVEF $(P<0.001)$. Additionally, isolated MV repair was performed in 354 (63.5\%) patients and preferred over combined procedures as the LVEF declined $(P=0.035)$. MV repair with concomitant cryoablation for AFIB was performed in 166 (29.8\%) patients. Moreover, concomitant cryoablation was more 
Table 2 Operative characteristics

\begin{tabular}{|c|c|c|c|c|c|}
\hline Variable & $\begin{array}{c}\text { Overall } \\
\text { LVEF }<50 \% \\
(n=557)\end{array}$ & $\begin{array}{c}\text { Group } 1 \\
\text { LVEF } 40 \%-49 \% \\
(n=294)\end{array}$ & $\begin{array}{c}\text { Group } 2 \\
\text { LVEF } 30 \%-39 \% \\
(n=145)\end{array}$ & $\begin{array}{c}\text { Group } 3 \\
\text { LVEF }<30 \% \\
(n=118)\end{array}$ & $P$ \\
\hline $\begin{array}{l}\text { Right-lateral mini-thoracotomy, } \\
n(\%)\end{array}$ & $327(58.7)$ & $200(68.0)$ & $82(56.6)$ & $45(38.1)$ & $<0.001$ \\
\hline Isolated MV repair, $n(\%)$ & $354(63.5)$ & $174(59.1)$ & $90(62.1)$ & $90(76.2)$ & 0.042 \\
\hline Additional cryoablation, $n(\%)$ & $166(29.8)$ & $100(34)$ & $44(30.1)$ & $22(18.6)$ & 0.009 \\
\hline Additional ASD closure, $n(\%)$ & $37(6.6)$ & $20(6.8)$ & $11(7.6)$ & $6(5.1)$ & 0.711 \\
\hline \multicolumn{6}{|l|}{ Annuloplasty ring model } \\
\hline CE Physio Ring, $n(\%)$ & 409 (73.4) & $206(70.1)$ & $99(68.3)$ & $104(88.1)$ & $<0.001$ \\
\hline CE Physio II Ring, $n(\%)$ & $29(5.2)$ & $26(8.8)$ & $1(0.7)$ & $2(1.7)$ & $<0.001$ \\
\hline IMR ETlogix Ring, $n(\%)$ & $60(10.7)$ & $28(9.5)$ & $23(15.9)$ & $9(7.6)$ & 0.008 \\
\hline $\begin{array}{l}\text { Cosgrove Annuloplasty Band, } \\
n(\%)\end{array}$ & $25(4.4)$ & $16(5.4)$ & $8(5.5)$ & $1(0.8)$ & 0.001 \\
\hline $\begin{array}{l}\text { MitralSolutions Annuloplasty } \\
\text { Band, } n(\%)\end{array}$ & $3(0.5)$ & $3(1.0)$ & $0(0)$ & $0(0)$ & - \\
\hline $\begin{array}{l}\text { SJM Flexible Annuloplasty Ring, } \\
n(\%)\end{array}$ & $5(0.9)$ & $2(0.7)$ & $3(2.1)$ & $0(0)$ & 0.655 \\
\hline SJM Rigid Saddle Ring, $n(\%)$ & $18(3.2)$ & $10(10.4)$ & $7(4.8)$ & $1(0.8)$ & 0.030 \\
\hline $\begin{array}{l}\text { SJM Seguin Annuloplasty Ring, } \\
n(\%)\end{array}$ & $8(1.4)$ & $3(1.0)$ & $4(2.8)$ & $1(0.8)$ & 0.417 \\
\hline $\mathrm{CPB}$ time, $\min$ & $111.7 \pm 39.4$ & $117.7 \pm 41.2$ & $106.3 \pm 34.7$ & $103.5 \pm 38.0$ & 0.001 \\
\hline Aortic cross-clamp time, min & $62.9 \pm 27.3$ & $68.6 \pm 28.5$ & $59.8 \pm 24.9$ & $52.4 \pm 23.6$ & $<0.001$ \\
\hline
\end{tabular}

ASD: atrial septal defect; CE: Carpentier-Edwards; CPB: cardiopulmonary bypass; IMR: ischemic mitral regurgitation; LVEF: left ventricular ejection fraction; MV: mitral valve; SJM: St. Jude Medical

Table 3 Perioperative characteristics

\begin{tabular}{|c|c|c|c|c|c|}
\hline Variable & $\begin{array}{c}\text { Overall } \\
\text { LVEF <50\% } \\
(n=557)\end{array}$ & $\begin{array}{c}\text { Group } 1 \\
\text { LVEF } 40 \%-49 \% \\
(n=294)\end{array}$ & $\begin{array}{c}\text { Group } 2 \\
\text { LVEF } 30 \%-39 \% \\
(n=145)\end{array}$ & $\begin{array}{c}\text { Group } 3 \\
\text { LVEF <30\% } \\
(n=118)\end{array}$ & $P$ \\
\hline Low cardiac output, $n(\%)$ & $24(4.3)$ & $8(2.7)$ & $5(3.4)$ & $11(9.3)$ & 0.010 \\
\hline $\mathrm{IABP}, n(\%)$ & $17(3.0)$ & $4(1.3)$ & $5(3.4)$ & $8(6.7)$ & 0.015 \\
\hline $\mathrm{ECMO}, n(\%)$ & $2(0.3)$ & $0(0)$ & $1(0.7)$ & $1(0.8)$ & 0.318 \\
\hline Myocardial infarction, $n(\%)$ & $4(0.7)$ & $0(0)$ & $3(2.1)$ & $1(0.8)$ & 0.053 \\
\hline New onset of AFIB, $n(\%)$ & $226(40.5)$ & $116(39.4)$ & $62(42.1)$ & $49(41.5)$ & 0.847 \\
\hline Reoperation due to bleeding, $n(\%)$ & $33(6)$ & $18(6.1)$ & $9(6.2)$ & $6(5.1)$ & 0.909 \\
\hline Major stroke, $n(\%)$ & $16(2.8)$ & $10(3.4)$ & $4(2.8)$ & $2(1.6)$ & 0.641 \\
\hline Sepsis, $n(\%)$ & $8(1.4)$ & $4(1.3)$ & $3(2.1)$ & $1(0.8)$ & 0.701 \\
\hline AKI on dialysis, $n(\%)$ & $20(3.5)$ & $9(3.1)$ & $7(4.8)$ & $4(3.3)$ & 0.640 \\
\hline Transfusion of blood units, $n$ & $1.7 \pm 4.8$ & $1.6 \pm 4.2$ & $2.2 \pm 6.5$ & $1.3 \pm 3.5$ & 0.275 \\
\hline Respiratory failure, $n(\%)$ & $54(9.7)$ & $23(7.8)$ & $23(15.9)$ & $8(6.7)$ & 0.013 \\
\hline 30-day mortality, $n(\%)$ & $18(3.2)$ & $4(1.4)$ & $5(3.4)$ & $9(7.6)$ & $<0.001$ \\
\hline
\end{tabular}

AFIB: atrial fibrillation; AKI: acute kidney injury; ECMO: extracorporeal membrane oxygenation; IABP: intra-aortic balloon pulsation; LVEF: left ventricular ejection fraction

frequently performed in patients with improved LVEF $(P=0.017)$. MV repair and ASD closure were performed in $37(6.6 \%)$ patients. Furthermore, CPB time and aortic cross-clamp time significantly shortened as the LVEF decreased across the groups $(P=0.002$ for CBP time; $P<0.001$ for the aortic cross-clamp time).

Among the three groups, most perioperative complications occurred with similar frequency (Table 3).
However, the low cardiac output (LCO) and postoperative intra-aortic balloon pulsation (IABP) implantation were significantly higher among patients in group $3(P=0.010$ for LCO; $P=0.015$ for IABP). Furthermore, the rates of respiratory failure and tracheostomy differed between groups.

Based on the degree of decreased LVEF, perioperative and long-term survival rates worsened (Table 3, Fig. 1A). 

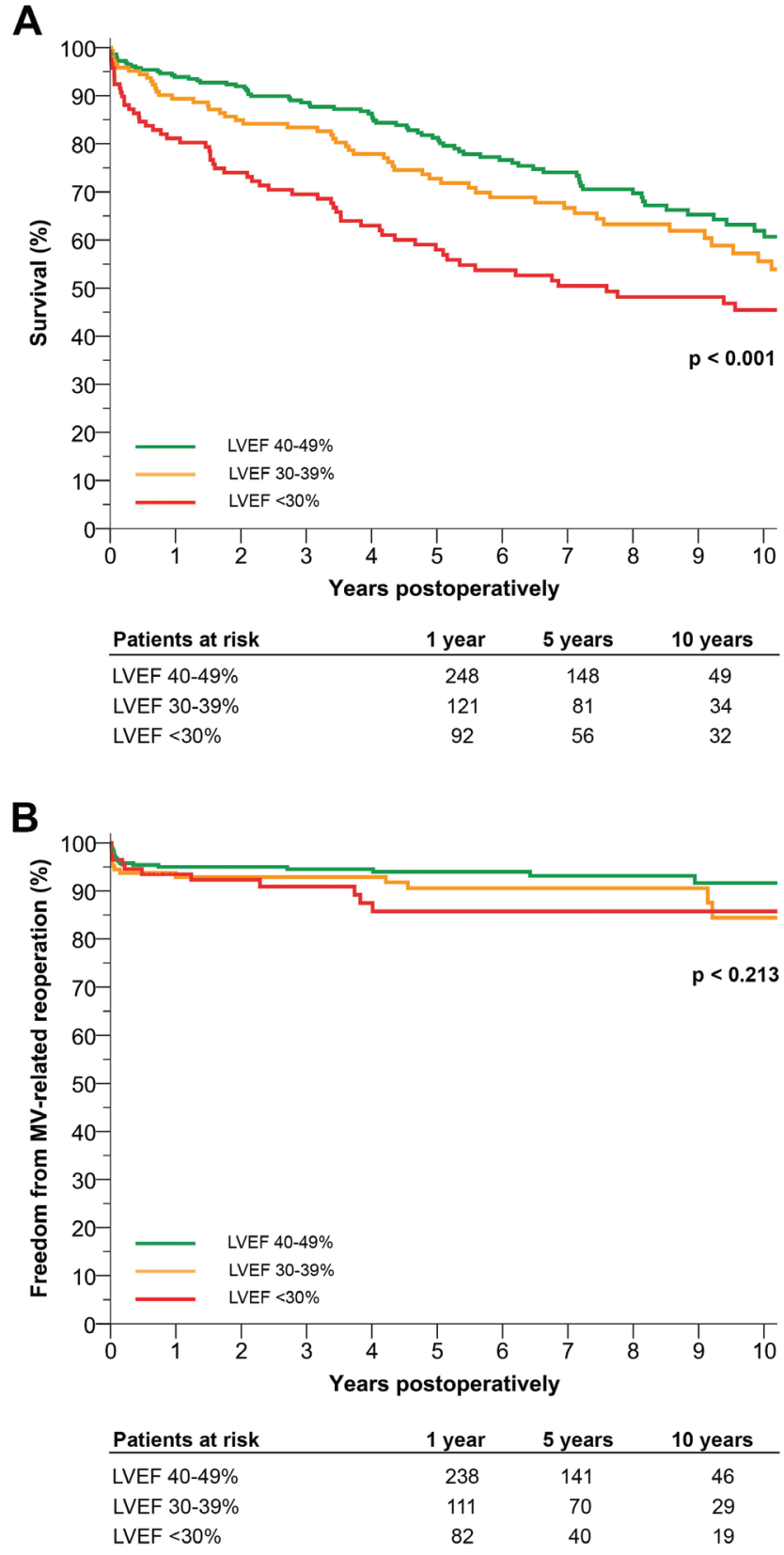

Fig. 1 The estimated survival (A) and freedom from mitral valve-related reoperation (B) after isolated mitral valve repair based on the study groups and study period. LVEF: left ventricular ejection fraction; MV: mitral valve

In groups 1-3, the 30-day mortality rate was $1.4 \%, 3.4 \%$, and $7.6 \%$, respectively $(P<0.001)$; estimated 1 -year survival rate was $93.9 \%, 89.4 \%$, and $82 \%$, respectively; estimated 5-year survival rate was $81.2 \%, 72.8 \%$, and $58 \%$, respectively; and estimated 10 -year survival rate was $63.2 \%, 55.6 \%$, and $45.4 \%$, respectively $(P<0.001)$ (Fig. 1A).
Risk factors for long-term survival were diabetes mellitus (hazard ratio [HR] 2.18, $P<0.001$ ), NYHA functional class III (HR 2.44, $P=0.038$ ) and class IV (HR 1.57, $P=0.038$ ), logistics EuroSCORE (HR 1.07, $P<0.001$ ), LCO (HR 2.15, $P=0.006$ ), acute kidney injury on dialysis (HR 2.70, $P=0.007$ ), and respiratory failure (HR 1.74, $P=0.043$ ) for the overall cohort (Table 4). Right-lateral mini-thoracotomy could be identified as factor for improvement of long-term survival (HR 0.60, $P=0.006$ ). LVEF was no significant risk factor for long-term survival.

Freedom from MV-related reoperation after 1 years was $95.8 \%, 92.9 \%$, and $93.5 \%$ for groups $1-3$, respectively; after the 5-year follow-up was $94 \%, 91.8 \%$, and $85.8 \%$ for groups $1-3$, respectively; while that after the 10-year follow-up was $91.6 \%, 84.4 \%$, and $85.8 \%$ for groups $1-3$, respectively $(P=0.213)$ (Fig. 1B).

During the entire follow-up, reoperation for any cause was required in 83 patients (14.9\%; Table 5); of these, $18(3.2 \%)$ patients underwent heart transplant, $11(2.0 \%)$ patients underwent left ventricular assist device implantation, and 37 (6.6\%) underwent MV replacement with optional concomitant procedures. The risk of any reoperation during the follow-up significantly increased $(P<0.001)$ as the LVEF declined, with the highest reoperation rate was observed in group 3.

\section{Discussion}

Owing to enhanced understanding of pathophysiological mechanisms of secondary MR, the use of contemporary MV repair techniques and the gain of experience in many centers of excellence for MV surgery, we hypothesized that the clinical outcomes after MV repair have improved in patients with decreased LVEF and MR, making it a treatment option for patients with increased operative risk. However, we widely recognize the decline of LVEF as a marker of increased operative risk and as a predictor of unfavorable clinical outcomes after MV repair. Hence, this study investigated the clinical outcomes after MV repair to elucidate the balance between risk and benefit for an enhanced clinical decision-making process in this patient cohort.

HF characterizes a heterogeneous group of patients whose prognosis varies markedly based on the severity of myocardial dysfunction. ${ }^{7)}$ Thus, the current guidelines highlight the significance of subdividing patients with HF based on the LVEF to optimize therapeutic options. The current HF guidelines consider three main groups of 
Table 4 Risk factors for long-term survival in patients with reduced left ventricular ejection fraction undergoing isolated mitral valve repair

\begin{tabular}{|c|c|c|c|c|c|}
\hline \multirow{2}{*}{ Variable } & \multirow{2}{*}{$n$} & \multirow{2}{*}{$\frac{\text { Univariate analysis }}{P \text { value }}$} & \multicolumn{3}{|c|}{ Multivariable Cox regression } \\
\hline & & & Hazard ratio & $95 \% \mathrm{CI}$ & $P$ value \\
\hline Mean age & 557 & 0.569 & - & - & - \\
\hline Female gender & 237 & 0.271 & - & - & - \\
\hline BMI & 557 & 0.785 & - & - & - \\
\hline Diabetes mellitus & 110 & 0.002 & 2.18 & $1.52-3.11$ & $<0.001$ \\
\hline Hypertension & 357 & 0.819 & - & - & - \\
\hline COPD & 29 & 0.347 & - & - & - \\
\hline PAD & 29 & 0.881 & - & - & - \\
\hline PHT & 108 & $<0.001$ & 0.98 & $0.94-1.02$ & 0.301 \\
\hline \multicolumn{6}{|l|}{ CAD } \\
\hline None & 465 & Reference & - & - & - \\
\hline 1-Vessel & 56 & 0.650 & - & - & - \\
\hline 2-Vessels & 22 & 0.665 & - & - & - \\
\hline 3-Vessels & 14 & 0.762 & - & - & - \\
\hline AFIB & 283 & 0.265 & - & - & - \\
\hline \multicolumn{6}{|l|}{ NYHA functional lass } \\
\hline $\mathrm{I} / \mathrm{II}$ & 251 & Reference & - & - & - \\
\hline III & 231 & 0.005 & 1.44 & $1.02-2.03$ & 0.038 \\
\hline IV & 75 & $<0.001$ & 1.57 & $1.03-2.40$ & 0.038 \\
\hline \multicolumn{6}{|l|}{ MR grade } \\
\hline Moderate-to-severe & 23 & Reference & - & - & - \\
\hline Severe & 534 & 0.530 & - & - & - \\
\hline \multicolumn{6}{|l|}{ LVEF } \\
\hline LVEF $40 \%-49 \%$ & 294 & Reference & - & - & - \\
\hline LVEF $30 \%-39 \%$ & 145 & 0.047 & 0.97 & $0.68-1.39$ & 0.868 \\
\hline $\mathrm{LVEF}<30 \%$ & 118 & 0.051 & 1.22 & $0.83-1.78$ & 0.306 \\
\hline \multicolumn{6}{|l|}{ Cardiomyopathy } \\
\hline Ischemic & 68 & Reference & - & - & - \\
\hline Non-ischemic dilated & 489 & 0.574 & - & - & - \\
\hline Logistic EuroSCORE & 557 & $<0.001$ & 1.07 & $1.05-1.10$ & $<0.001$ \\
\hline Right-lateral mini-thoracotomy & 372 & $<0.001$ & 0.60 & $0.42-0.86$ & 0.006 \\
\hline Additional cryoablation & 166 & $<0.001$ & 0.77 & $0.54-1.09$ & 0.143 \\
\hline Additional ASD closure & 37 & 0.050 & - & - & - \\
\hline CPB time & 557 & 0.054 & - & - & - \\
\hline Aortic cross-clamp time & 557 & 0.053 & - & - & - \\
\hline Low cardiac output & 24 & 0.007 & 2.15 & $1.24-3.73$ & 0.006 \\
\hline IABP & 17 & 0.054 & - & - & - \\
\hline ECMO & 2 & 0.063 & - & - & - \\
\hline Myocardial infarction & 4 & 0.578 & - & - & - \\
\hline New onset of AFIB & 226 & 0.690 & - & - & - \\
\hline Reoperation due to bleeding & 33 & 0.145 & - & - & - \\
\hline Major stroke & 16 & 0.099 & - & - & - \\
\hline Sepsis & 8 & 0.430 & - & - & - \\
\hline AKI on dialysis & 20 & $<0.001$ & 2.70 & $1.31-5.58$ & 0.007 \\
\hline Transfusion of RBC & 198 & 0.057 & - & - & - \\
\hline Respiratory failure & 54 & 0.032 & 1.74 & $1.02-2.98$ & 0.043 \\
\hline
\end{tabular}

AFIB: atrial fibrillation; AKI: acute kidney injury; ASD: atrial septal defect; BMI: body mass index; CAD: coronary artery disease; CI: confidence interval; COPD: chronic obstructive pulmonary disease; CPB: cardiopulmonary bypass; ECMO: extracorporeal membrane oxygenation; IABP: intra-aortic balloon pulsation; LVEF: left ventricular ejection fraction; MR: mitral regurgitation; NYHA: New York Heart Association; PAD: peripheral arterial disease; PHT: pulmonary hypertension; RBC: red blood cells 
Table 5 Incidence and causes of reoperations

\begin{tabular}{lccccc}
\hline Variable & $\begin{array}{c}\text { Overall } \\
\text { LVEF }<50 \% \\
(n=557)\end{array}$ & $\begin{array}{c}\text { Group 1 } \\
\text { LVEF 40\%-49\% } \\
(n=294)\end{array}$ & $\begin{array}{c}\text { Group 2 } \\
\text { LVEF 30\%-39\% } \\
(n=145)\end{array}$ & $\begin{array}{c}\text { Group 3 } \\
\text { LVEF <30\% } \\
(n=118)\end{array}$ & $P$ \\
\hline $\begin{array}{l}\text { Reoperation, } n(\%) \\
\text { Causes for reoperation: }\end{array}$ & $83(14.9)$ & $27(9.2)$ & $24(16.6)$ & $32(27.1)$ & $<0.001$ \\
$\quad$ MV re-repair, $n(\%)$ & $10(1.8)$ & $3(1.0)$ & $3(2.1)$ & $4(3.4)$ & 0.905 \\
MV re-repair + concomitant & & & & & - \\
procedures, $n$ (\%) & $1(0.2)$ & $0(0)$ & $1(0.7)$ & $0(0)$ & - \\
MV replacement, $n(\%)$ & $25(4.5)$ & $12(4.1)$ & $8(5.5)$ & $5(4.2)$ & 0.228 \\
MV replacement + & & & & $3(2.5)$ & 0.174 \\
concomitant procedures, $n(\%)$ & $12(2.2)$ & $7(2.4)$ & $2(1.4)$ & $0(0)$ & - \\
TV repair, $n$ (\%) & $1(0.2)$ & $1(0.3)$ & $0(0)$ & $12(10.2)$ & 0.157 \\
Heart transplant, $n(\%)$ & $18(3.2)$ & $0(0)$ & $6(4.1)$ & $8(6.8)$ & 0.020 \\
LVAD implantation, $n(\%)$ & $11(2.0)$ & $1(0.3)$ & $2(1.4)$ & $0(0)$ & 0.655 \\
Other cardiac surgery, $n(\%)$ & $5(0.9)$ & $3(1.0)$ & $2(1.4)$ & & \\
\hline
\end{tabular}

LVAD: left ventricular assist device; LVEF: left ventricular ejection fraction; MV: mitral valve; TV: tricuspid valve

patients based on the $\mathrm{LVEF}^{7}$; its differentiation is crucial because of different underlying etiologies, symptoms, age, associated conditions (e.g., AFIB and coronary artery disease), clinically relevant comorbidities, and response to therapies. ${ }^{6}{ }^{615)}$ As an $\mathrm{LVEF}<40 \%$ remains a vast range, we presented our results by subdividing patients with HFrEF into two more subgroups, as described above. Furthermore, HF can be related to clinically relevant secondary MR. However, the optimal surgical strategies for the management of secondary MR in patients with impaired LVEF remain debatable. ${ }^{8,16,17)}$ Reportedly, undersized ring annuloplasty in consideration of predictors for MV repair failure is the preferred surgical repair strategy ${ }^{18)}$; nevertheless, residual or recurrent MR are limitations of this approach and related to higher rate of cardiac events and reduced survival. ${ }^{16)} \mathrm{MV}$ replacement averts the risk of residual or recurrent MR but is not related to enhanced survival compared with MV repair. ${ }^{8)}$ To date, several studies have reported that MV repair offers a better short- and long-term survival compared with MV replacement in patients with non-ischemic dilated or ischemic cardiomyopathy. ${ }^{16,17)}$

In this study, patients' preoperative characteristics in the three study groups were similar, and baseline pathologies were equally distributed across the groups. Only the logistic EuroSCORE markedly increased from groups 1 to 3, the natural consequence of LVEF worsening and HF. Additionally, patients with a more reduced LVEF (groups 2 and 3) were markedly more symptomatic than patients from group 1 based on the NYHA functional class.

In all groups, most patients presented non-ischemic dilatated cardiomyopathy, and only a minority reported ischemic cardiomyopathy; this contradicts De Bonis et al. ${ }^{16)}$ In their study, $33 \%$ of patients presented non-ischemic dilated cardiomyopathy and $67 \%$ ischemic cardiomyopathy; moreover, they treated patients with MR by MV repair whenever possible. ${ }^{16)}$ In this study, the number of patients with MR and ischemic cardiomyopathy treated by MV repair elevated with the surgical experience over the past 20 decades.

Intraoperatively, we noted a tendency to simplify MV repair procedures as the LVEF decreased, explaining how both CPB and aortic cross-clamp times markedly decreased from groups 1 to 3 . Similarly, concomitant procedures, such as cryoablation procedure, or more sophisticated surgical approaches, such as right mini-thoracotomy for minimally invasive cardiac surgery, markedly became less frequent as the LVEF reduced. Furthermore, isolated MV repair was preferred for patients with the most depressed LVEF.

Our postoperative follow-up revealed that 30-day mortality and mid-/long-term survival were markedly affected by the LVEF worsening. However, large series with general population patients have reported a 30-day mortality of $0.2 \%-2.6 \%$, corroborating group 1 in our study. ${ }^{12,19,20)}$ Based on the observed 30-day mortality in the present study, surgical mortality was better as expected by the logistics EuroSCORE, especially in groups 1 and 2. This can be explained by the ongoing improvement in surgical MV repair with improved repair techniques and greater experience of MV repair surgeons. ${ }^{21)}$

In general population patients undergoing minimally invasive MV repair, we previously reported the survival rates of $87.0 \% \pm 0.7 \%$ and $74.2 \% \pm 1.4 \%$ at 5 and 
10 years, respectively. ${ }^{12,22)}$ Additionally, freedom from reoperation was $96.6 \% \pm 0.4 \%$ and $92.9 \% \pm 0.9 \%$ at 5 and 10 years, respectively. ${ }^{12)}$ However, these outcomes could be attributed to the follow-up of patients who are otherwise healthy, with a long-life expectancy and mostly without a major structural cardiac compromise.

To date, only limited studies have addressed the same clinical question. Investigating MV repair in functional MR, De Bonis et al. reported a 2.5-year survival of $92 \%$ (mean LVEF, 31\%), ${ }^{16)}$ which is consistent with group 2 in this study that exhibits a lower 2.5-year survival rate of $84.2 \%$ (Fig. 1A). Perhaps, the high rate of severe MR $(96 \%)$ in group 2 as an index for the disease severity could explain the difference.

Nevertheless, the observed clinical outcomes in this HF cohort could be considered as acceptable, as most of our patients presented severe MR, and many of those experienced highly severe HF with a grim prognosis and a poor quality of life. Thus, the results of this high-risk patient cohort are acceptable compared with prior literature in the normal population. ${ }^{12,14,19,20)}$ As many of our patients received MV repair as a bridge to transplant or ventricular assist devices implantation, they were expected to sooner or later undergo reoperation.

Across all the study groups, the frequency of postoperative complications was quite similar. Respiratory failure and tracheostomy markedly increased from groups $1-2$ and unexpectedly decreased their frequency in group 3; the explanation for this contradictory tendency is not associated with HF-dependent factors but the decision to limit therapeutic efforts in patients displaying poor prognosis.

Diabetes mellitus, NYHA functional class III and IV, and logistic EuroSCORE were identified as preoperative risk factors for long-term survival. Similarly, the NYHA functional class has also been identified as predictor for late mortality in previous investigations. ${ }^{23)}$ The preoperative LVEF was no significant risk factor for long-term survival and underlines that LVEF must be discussed with the other preoperative factors for surgical decision-making. The appearance of perioperative LCO, acute kidney injury on dialysis, and respiratory failure are associated with worse long-term outcome for MV surgery in this patient cohort (see also Table 4). For these reasons, best perioperative intensive care is mandatory and can improve significantly the long-term outcome in this critical patient cohort. Surprisingly, minimally MV repair via a right-sided minithoracotomy significantly reduces the risk for long-term mortality (HR 0.6, $P=0.006$ ). Besides the well-known advantages and disadvantages of the minimally invasive approach, ${ }^{14,22)}$ the reasons for this effect in the present study are speculative. The surgical approach was selected patient specifically by the cardiac surgeon. It is known that full sternotomy is the favorable approach in patients with elevated operative risk, which could be a bias in this analysis. Contrariwise, we can summarize that the minimally invasive MV repair can be performed safely and can be recommended in these patients, if technically possible. ${ }^{22}$ )

A trend was found for an increase in MV-related reoperation in groups 2 (freedom from MV-related reoperation: $91.8 \%$ ) and 3 (freedom from MV-related reoperation: $85.8 \%$ ) after 5 years, but the difference between all groups was non-significant. These differences between the groups can be explained by a higher rate of residual MR due to progression of ischemic or non-ischemic cardiomyopathy in patients with reduced LVEF. ${ }^{24)}$

Of note, surgical MV repair must be compared with currently available percutaneous repair techniques. In the EVEREST II study, percutaneous devices less effectively reduced MR compared with conventional surgery, as patients in the MitraClip group required more frequent reoperations after 12 months. ${ }^{25,26)}$ Reportedly, superior safety correlated with the percutaneous procedure. ${ }^{25,26)}$ However, the mean Society of Thoracic Surgeons (STS) score of the EVEREST II population was 5\%, which does not denote a risk as high as that of patients enrolled in this study. Additionally, patients included in the transcatheter mitral valve interventions (TRAMI) registry exhibited a marked improvement in symptoms and functional class at 1 year after MitraClip ${ }^{27}$; nevertheless, 1-year mortality was $20.3 \%$, higher than 1-year mortality observed in this study. In the TRAMI registry population, NYHA class IV, anemia, previous $\mathrm{AV}$ intervention, serum creatinine $\geq 1.5 \mathrm{mg} / \mathrm{dL}$, peripheral artery disease, $\mathrm{LVEF}<30 \%$, severe tricuspid regurgitation, and procedural failure were recognized as predictors of 1-year mortality. Moreover, the failure of procedural success displayed the highest HR regarding the prediction of 1-year mortality. The knowledge of these predictors facilitates multidisciplinary discussion to enable the appropriate selection of patients who could benefit from surgical MV repair as the first-line approach. Furthermore, patients included in the TRAMI registry exhibited a very high logistic EuroSCORE (mean logistic EuroSCORE, 20\%) or were markedly older than our study patients.

\section{Clinical implications}

Although prior studies have considered HF as a homogeneous patient population with reduced LVEF, those did 
not provide any further specification and, thus, reported suboptimal clinical outcomes after MV repair.,2,6,18) Nevertheless, if patients are precisely classified based on the current guidelines, decision-making and patient selection for MV repair turn better, and clinical outcomes improve. Moreover, the data underline the vital role of surgical MV repair in patients with impaired LVEF. Furthermore, LVEF alone should not be used as a parameter for therapeutic decision-making in this cohort.

\section{Limitations}

One of the major limitations of this study is its retrospective nature and the resultant issues related thereto. Another limitation is the failure to enroll patients in whom a repair was attempted but then converted to an MV replacement procedure. Finally, patients with HFpEF were not enrolled in this study and, thus, outcomes in this unique patient population remain unclear.

\section{Conclusions}

This study establishes that MV repair could be performed with low operative mortality and good-to-acceptable mid- and long-term results in patients with reduced LVEF and MR. Moreover, perioperative and long-term mortality increase as the preoperative LVEF decreases.

\section{Disclosure Statement}

Michael Borger has received speaker honoraria from Edwards Lifesciences, Medtronic, LivaNova, and CryoLife. All other authors have no conflict of interest related to the present original article.

\section{References}

1) Baumgartner H, Falk V, Bax JJ, et al. 2017 ESC/EACTS guidelines for the management of valvular heart disease. Eur Heart J 2017; 38: 2739-91.

2) Nishimura RA, Otto CM, Bonow RO, et al. $2017 \mathrm{AHA} /$ ACC focused update of the 2014 AHA/ACC guideline for the management of patients with valvular heart disease: a report of the American College of Cardiology/ American Heart Association Task Force on Clinical Practice Guidelines. J Am Coll Cardiol 2017; 70: 252-89.

3) Mirabel M, Iung B, Baron G, et al. What are the characteristics of patients with severe, symptomatic, mitral regurgitation who are denied surgery? Eur Heart J 2007; 28: $1358-65$.

4) Asgar AW, Mack MJ, Stone GW. Secondary mitral regurgitation in heart failure: pathophysiology, prognosis, and therapeutic considerations. J Am Coll Cardiol 2015; 65: $1231-48$.

5) Wang TJ, Evans JC, Benjamin EJ, et al. Natural history of asymptomatic left ventricular systolic dysfunction in the community. Circulation 2003; 108: 977-82.

6) O'Gara PT, Grayburn PA, Badhwar V, et al. 2017 ACC expert consensus decision pathway on the management of mitral regurgitation: a report of the American College of Cardiology Task Force on expert consensus decision pathways. J Am Coll Cardiol 2017; 70: 2421-49.

7) Ponikowski P, Voors AA, Anker SD, et al. 2016 ESC guidelines for the diagnosis and treatment of acute and chronic heart failure: the task force for the diagnosis and treatment of acute and chronic heart failure of the European Society of Cardiology (ESC) developed with the special contribution of the Heart Failure Association (HFA) of the ESC. Eur Heart J 2016; 37: 2129-200.

8) Acker MA, Parides MK, Perrault LP, et al. Mitral-valve repair versus replacement for severe ischemic mitral regurgitation. N Engl J Med 2014; 370: 23-32.

9) Fattouch K, Guccione F, Sampognaro R, et al. POINT: efficacy of adding mitral valve restrictive annuloplasty to coronary artery bypass grafting in patients with moderate ischemic mitral valve regurgitation: a randomized trial. J Thorac Cardiovasc Surg 2009; 138: 278-85.

10) Goldstein D, Moskowitz AJ, Gelijns AC, et al. Twoyear outcomes of surgical treatment of severe ischemic mitral regurgitation. N Engl J Med 2016; 374: 344-53.

11) $\mathrm{Wu} \mathrm{AH}$, Aaronson $\mathrm{KD}$, Bolling SF, et al. Impact of mitral valve annuloplasty on mortality risk in patients with mitral regurgitation and left ventricular systolic dysfunction. J Am Coll Cardiol 2005; 45: 381-7.

12) Davierwala PM, Seeburger J, Pfannmueller B, et al. Minimally invasive mitral valve surgery: "The Leipzig experience". Ann Cardiothorac Surg 2013; 2: 744-50.

13) Vollroth M, Seeburger J, Garbade J, et al. Conversion rate and contraindications for minimally invasive mitral valve surgery. Ann Cardiothorac Surg 2013; 2: 853-4.

14) Seeburger J, Borger MA, Falk V, et al. Minimal invasive mitral valve repair for mitral regurgitation: results of 1339 consecutive patients. Eur J Cardiothorac Surg 2008; 34: 760-5.

15) Butler J, Fonarow GC, Zile MR, et al. Developing therapies for heart failure with preserved ejection fraction: current state and future directions. JACC Heart Fail 2014; 2: 97-112.

16) De Bonis M, Ferrara D, Taramasso M, et al. Mitral replacement or repair for functional mitral regurgitation in dilated and ischemic cardiomyopathy: is it really the same? Ann Thorac Surg 2012; 94: 44-51.

17) Vassileva CM, Boley $T$, Markwell S, et al. Metaanalysis of short-term and long-term survival following repair versus replacement for ischemic mitral regurgitation. Eur J Cardiothorac Surg 2011; 39: 295-303.

18) De Bonis M, Al-Attar N, Antunes M, et al. Surgical and interventional management of mitral valve regurgitation: a position statement from the European Society 
of Cardiology Working Groups on Cardiovascular Surgery and Valvular Heart Disease. Eur Heart J 2016; 37: 133-9.

19) Glauber M, Miceli A, Canarutto D, et al. Early and long-term outcomes of minimally invasive mitral valve surgery through right minithoracotomy: a 10-year experience in 1604 patients. J Cardiothorac Surg 2015; 10: 181.

20) McClure RS, Athanasopoulos LV, McGurk S, et al. One thousand minimally invasive mitral valve operations: early outcomes, late outcomes, and echocardiographic follow-up. J Thorac Cardiovasc Surg 2013; 145: 1199-206.

21) Suri RM, Clavel MA, Schaff HV, et al. Effect of recurrent mitral regurgitation following degenerative mitral valve repair: long-term analysis of competing outcomes. J Am Coll Cardiol 2016; 67: 488-98.

22) Garbade J, Seeburger J, Merk DR, et al. Mitral valve pathology in severely impaired left ventricles can be successfully managed using a right-sided minimally invasive surgical approach. Eur J Cardiothorac Surg 2013; 44: e1-7.

23) Meyer MA, von Segesser LK, Hurni M, et al. Longterm outcome after mitral valve repair: a risk factor analysis. Eur J Cardiothorac Surg 2007; 32: 301-7.

24) Magne J, Sénéchal M, Dumesnil JG, et al. Ischemic mitral regurgitation: a complex multifaceted disease. Cardiology 2009; 112: 244-59.

25) Feldman T, Foster E, Glower DD, et al. Percutaneous repair or surgery for mitral regurgitation. $\mathrm{N}$ Engl $\mathrm{J}$ Med 2011; 364: 1395-406.

26) Feldman T, Kar S, Elmariah S, et al. Randomized comparison of percutaneous repair and surgery for mitral regurgitation: 5-year results of EVEREST II. J Am Coll Cardiol 2015; 66: 2844-54.

27) Puls M, Lubos E, Boekstegers P, et al. One-year outcomes and predictors of mortality after MitraClip therapy in contemporary clinical practice: results from the German transcatheter mitral valve interventions registry. Eur Heart J 2016; 37: 703-12. 